\title{
The Relationships between Cognitive Neuropsychological State, Oxidative Stress and Genotoxicity in Radiology Staff
}

\author{
Hajar Akbari Dastjerdi', Mohammad Fazilati' ${ }^{1}$, Ali Akbar Malekirad ${ }^{1,2 *}$, \\ Habibollah Nazem1, Mohammad Abdollahi'2, Akbar Zerratpisheh ${ }^{3}$ \\ ${ }^{1}$ Department of Biology, Payame Noor University, Tehran, Iran \\ ${ }^{2}$ Faculty of Pharmacy, and Pharmaceutical Sciences Research Center, Tehran University of Medical Science \\ (TUMS), Tehran, Iran \\ ${ }^{3}$ Islamic Azad University, Jiroft Branch, Jiroft, Iran \\ Email: AK malekirad@yahoo.com, Malekirad1973@gmail.com, malekirad@Tabrizu.ac.ir
}

Received 25 April 2014; revised 27 May 2014; accepted 4 June 2014

Copyright (C) 2014 by authors and Scientific Research Publishing Inc.

This work is licensed under the Creative Commons Attribution International License (CC BY).

http://creativecommons.org/licenses/by/4.0/

c) (i) Open Access

\section{Abstract}

Imbalance between the production and removal of reactive oxygen species (ROS) has been used for characterizing Oxidative stress. Extra amount of ROS are hazardous and can lead damages to the cells resulting in harsh diseases. For cancer, diabetes, psychological disorders and age-related disorders, latent of ROS in induction of alteration and DNA harm is a critical factor. Regarding these finding, the current research was planned to test the relationship between cognitive neuropsychological state and oxidative markers and genotoxicity in radiology staff. Total Number of 47 radiology staff, working in the radiology center of a referral University Hospital in Shiraz, were invited in the study. The participants were occupationally exposed to low-dose ionizing radiation (x-ray) for more than 2 years. Psychological disorder was analyzed by SCL-90-R. In the plasma, lipid peroxidation, DNA harm, catalase, superoxide dismutase, myeloperoxidase, and glutathione peroxidase activity were calculated. Associations between parameters were measured by using Pearson correlation coefficient and multivariate linear regression analyses. As a result, there was a positive considerable connection between catalase and paranoid ideation, Obsessive-Compulsive, Anxiety and total. Furthermore, the correlation between psychoticism, Interpersonal Sensitivity, Paranoid Ideation and DNA damage was positive. There were positive relationships between superoxide dismutase and psychomotor speed, initiative/energy, selective attention and verbal memory. So, there were significant relationships among interpersonal sensitivity, somatization, depression and gender. But, there were no major relationships among psychological distress, history workers and oxidative stress. In so many words, Oxidative stress and genotoxicity result in psychological disorders in radiology staff. So, radiology staff should have more attention, 
as they suffered from some psychological disorders.

Keywords

Poisoning, Ionizing Radiation, Cognitive Neuropsychological Stress, Oxidative Stress

\section{Introduction}

It would be mentioned that when there is no balance between the production of free radicals and reactive oxygen species (ROS), oxidative stress is created and antioxidant defense system is consisted of antioxidant enzymes like superoxide dismutase of copper, zinc and, manganese, and small molecule antioxidants such as glutathione, vitamin E and vitamin C. Some free radicals such as nitrogen reactive species (RNS) and reactive oxygen species (ROS) lead oxidative damage to RNA DNA lipids and proteins [1].

A lot of studies propose the effect of oxidative stress on people's health, because it can cause diabetes, cancer, Parkinson's disease and cardiovascular diseases [2]-[4]. In fact, as oxidative stress is one of reasons in hurting to brain, so many researchers were moved to survey the cognitive and psychological troubles of groups who are at risk. As a reason, consuming a lot of oxygen by brain, as well as, the extensive amounts of iron and copper catalyzes free radical reactions.

As a matter of fact, utilizing oxygen in people at risk raised to 1.5 times and approximately $2 \%$ of the oxygen is changed tore active oxygen species (ROS), this oxygen is used by cells in the procedure of oxidative phosphorylation [5].

Through the disturbance in brain's operation and as a result of reduced antioxidant defense against lipid per oxidation and oxidative stress, improved oxidative stress can cause mental disorders.

Oxidative stress can cause to some diseases and disorders as DSM-IV-TR diagnostic criteria has mentioned: delusional disorders, mental retardation, autistic disorder, attention deficit, hyperactivity, delirium, dementia, disorders related to alcohol, opioid-related disorders, sexual impotence, amphetamines (or amphetamine-like) related disorders, sleep disorders, nicotine dependence disorders, schizophrenia, mood disorders, anxiety disorders and eating disorders [6]. In other word, many researchers reported the impacts of oxidative stress on each psychological disorder. For instance, major depressive disorder (MDD) is one of the results of raised lipid peroxidation [7] [8] and based on an investigation by Sarandol et al. (2007), whose signs had still remained in patients until six weeks of curing [9].

Moreover, in bipolar disorder (BD) due to producing extremes (ROS) such as hydroxyl radicals and by harming DNA structure, oxidative stress has played a major role [10]. Some studies indicated that many anxiety disorders like: social phobia (SP), symptoms of severe anxiety, obsessive-compulsive disorder (OCD), frustration and the indiscriminate use of alcohol has been created by enlarged level so $\mathrm{f}$ lipid peroxidation [11]-[14].

In one study by for Lenza and Miller (2006) reviewed that DNA damage can lead to anxiety disorders [15]. Another disease which can be created by oxidative stress is Autism. In comparative study on two groups of autistic patients and control groups, result has been revealed that in autistic patients, decline in the level of endogenous antioxidants and mitochondrial dysfunction were more than control groups [16] [17].

On the other hand, cognitive dysfunction and reduced antioxidant capacity have good correlation with each other. One of risk factors for cognitive impairment can be antioxidant deficiency or enlarged levels of oxidative stress. To be honest, one of main cognitive results of oxidative stress and diminish the cognitive function is Alzheimer's disease [18]. Job stress as one of various types of psychological stress can raise symptoms of mental disorders. Gil et al. (2002) detected that premature aging can be created as a result of relationship between oxidative stress and psychological stress [19].

After pointing to some researchers in oxidative stress [1] and the effect of medicinal plants on oxidative stress in radiology staff [2] [4], researchers would like to conduct a research on the interaction between oxidative stress and cognitive neuropsychological disorders. So the main purpose of this study is to distinguish the relationship between cognitive neuropsychological state and oxidative markers and genotoxicity in radiology staff.

\section{Materials and Methods}

The materials which were applied in this research are as follow: Tris base, tetraethoxy propane (MDA), 
2-thiobarbituric acid (TBA), trichloroacetic acid (TCA), n-butanol, dithiobis-2-nitrobenzoic acid (DTNB), 2,4,6tripyridyl-striazine (TPTZ) from Sigma-Aldrich Chemie (Gmbh Munich, Germany), $\mathrm{HNO}_{3}, \mathrm{H}_{2} \mathrm{SO}_{4}, \mathrm{HCL}, \mathrm{NaCl}$, $\mathrm{PdCl}_{2}$ from Merck Chemical Co. (Darmstadt, Germany), and ELISA kits for oxidative stress biomarkers and DNA damage from Cayman Chemical Co. (Michigan, USA).

\section{Subjects}

The initial sample of this study consisted of 47 subjects and they were 19 males and 28 females. Before starting the study, all participants gave informed consent and the agreement of the study protocol by the Institute Review Committee. It is worth mentioning that the participants were consisted of 47 Shiraz hospital employees of Radiology staff in 2013. The necessary criteria included: there should not any experience in taking drugs, smoking, alcohol, antioxidants. On the other hand; they shouldn't have special diseases such as cancer, diabetes, cardiovascular, thyroid disorder and respiratory disorders.

Another certain situation for participant was that they had more than two years of work experience. So, they informed orally and in written form about the purpose of this test. In this time, they announced their agreement for taking part in this study. During the investigation due to questionnaire, was reveal that any of staff employed in the Department of Radiology had not been occupationally exposed to any other destructive agents except the risk of radiation and in the 12 months prior to the study. Therefore, the result indicated that they had no history of radiation assisted diagnosis and radiation therapy. The percent consumption of alcohol, specific drugs, cigarettes and other tobacco products was zero and all of them were healthy. Through Scl90 and Cognitive neuropsychological impairment which were assessed by SNI questionnaire, psychological distress and psychological symptoms had been analyzed.

By employing nine primary symptom dimensions and three summary scores termed global scores, the SCL-90$\mathrm{R}$ evaluated psychological distress.

Somatization (SOM), Obsessive-Compulsive (OBS), Hostility (HOS), Interpersonal Sensitivity (INT), Depression (DEP), Psychoticism (PSY) Anxiety (ANX), Phobic Anxiety (PHOB), Paranoid Ideation (PAR), and were labeled as principal symptom.

The Global Severity Index (GSI), the Positive Symptom Distress Index (PSDI), and the Positive Symptom Total (PST) were as global measures [20] [21].

In order to identify high-risk subjects for neurocognitive impairment, the Iranian version of the SNI was used [22]. The SNI composed of seventy-six pieces which has regarding on daily memory and attention glitches is the self-report questionnaire. Appling this questionnaire helps to distinguish self-experienced deficits of attention [23] [24].

\section{Assay of Oxidative Stress Markers}

As DNA damage and glutathione peroxidase (GPX) were analyzed and searched in our previous study by means of ELISA kits, the activities of superoxide dismutase (SOD), 8-hydroxy-2-deoxy guano sine (8-OH-dG), catalase (CAT) and meyleoperoxidase [MPO]were measured [2]. TBA test was applied [25]. For the measuring of lipid peroxidation TBA test was applied [25]. The total antioxidant capacity (TAC) was measured in order to analyze the capability of plasma in declining $\mathrm{Fe}^{3+}$ to $\mathrm{Fe}^{2+}$ by existing TPTZ. So, $\mathrm{Fe}^{2+}-\mathrm{TPTZ}$ is absorbed as blue complex at $593 \mathrm{~nm}$ [26]. Through estimating the plasma total thiol molecules (TTM) DTNB has been used. DTNB forms a yellow complex which has well absorbance at $412 \mathrm{~nm}$ [27].

For surviving in the association between variables, Pearson coefficient was used. Data were accessible as mean \pm SD. p values lower than 0.05 were considered statistically significant. Stats Direct 2.7.9 was used to analyze data.

\section{Results}

As indicated in Table 1 has shown that average levels of subject's ages, sex and years of exposure. Data analysis revealed a major positive relationship between CAT in one hand and OBS $(\mathrm{R}=0.337, \mathrm{p}=0.025), \mathrm{ANX}(\mathrm{R}=$ 0.345, $\mathrm{p}=0.018)$, PAR $(\mathrm{R}=0.375, \mathrm{p}=0.009)$ and total $(\mathrm{R}=0.326, \mathrm{p}=0.020)$ on the other hand. In addition, the correlation between 8-OH-dG on one side and INT $(\mathrm{R}=0.364, \mathrm{p}=0.012)$, PAR $(\mathrm{R}=0.305, \mathrm{p}=0.037)$ and PSY $(\mathrm{R}=0.389, \mathrm{p}=0.007)$ on the other side were positive as, reader can observe in Table 2 . Table 3 has re- 
Table 1. The result of demographic data in workers.

\begin{tabular}{ccccc}
\hline & Age (years) & Sex & Work history (year) & Exposure history (year) \\
\hline Workers & $28.57 \pm 6.59$ & $47(19$ male; 28 female $)$ & $9.77 \pm 7.19$ & $12.40 \pm 10.45$ \\
\hline
\end{tabular}

Data represent mean \pm SD.

Table 2. The correlation between oxidative stress markers and psychological distress in radiology staff.

\begin{tabular}{|c|c|c|c|c|c|c|c|c|c|}
\hline 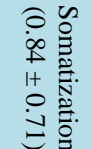 & 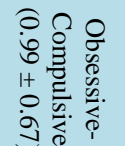 & 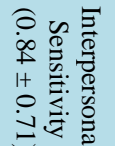 & 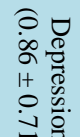 & 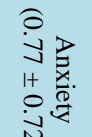 & 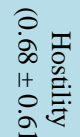 & 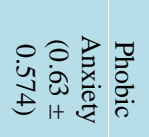 & 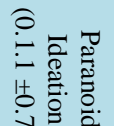 & 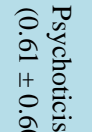 & $\vec{\theta}$ \\
\hline
\end{tabular}

\begin{tabular}{|c|c|c|c|c|c|c|c|c|c|c|}
\hline $\begin{array}{c}\text { LPO } \\
(6.43 \pm 1.81 \\
\mu \mathrm{M})\end{array}$ & $\begin{array}{l}R=0.042 \\
p=0.780\end{array}$ & $\begin{array}{c}\mathrm{R}=-0.015 \\
\mathrm{p}=0.920\end{array}$ & $\begin{array}{c}\mathrm{R}=-0.177 \\
\mathrm{p}=0.234\end{array}$ & $\begin{array}{c}\mathrm{R}=-0.089 \\
\mathrm{p}=0.554\end{array}$ & $\begin{array}{c}\mathrm{R}=-0.026 \\
\mathrm{p}=0.861\end{array}$ & $\begin{array}{l}\mathrm{R}=0.102 \\
\mathrm{p}=0.493\end{array}$ & $\begin{array}{c}\mathrm{R}=-0.0306 \\
\mathrm{p}=0.843\end{array}$ & $\begin{array}{c}\mathrm{R}=-0.016 \\
\mathrm{p}=0.917\end{array}$ & $\begin{array}{l}R=0.060 \\
p=0.687\end{array}$ & $\begin{array}{c}\mathrm{R}=-0.032 \\
\mathrm{p}=0.831\end{array}$ \\
\hline $\begin{array}{c}\text { MPO } \\
(7.94 \pm 9.36 \\
\mu \mathrm{M})\end{array}$ & $\begin{array}{c}\mathrm{R}=-0.005 \\
\mathrm{p}=0.971\end{array}$ & $\begin{array}{c}\mathrm{R}=-0.148 \\
\mathrm{p}=0.322\end{array}$ & $\begin{array}{c}\mathrm{R}=-0.241 \\
\mathrm{p}=0.102\end{array}$ & $\begin{array}{c}\mathrm{R}=-0.182 \\
\mathrm{p}=0.221\end{array}$ & $\begin{array}{c}\mathrm{R}=-0.134 \\
\mathrm{p}=0.367\end{array}$ & $\begin{array}{l}R=0.015 \\
p=0.921\end{array}$ & $\begin{array}{c}\mathrm{R}=-0.208 \\
\mathrm{p}=0.161\end{array}$ & $\begin{array}{c}\mathrm{R}=-0.164 \\
\mathrm{p}=0.271\end{array}$ & $\begin{array}{c}R=-0.252 \\
p=0.087\end{array}$ & $\begin{array}{c}2 \mathrm{R}=-0.158 \\
\mathrm{p}=0.289\end{array}$ \\
\hline $\begin{array}{c}\text { SOD } \\
(0.65 \pm 0.29 \\
\mathrm{U} / \mathrm{mg} \text { protein })\end{array}$ & $\begin{array}{l}\mathrm{R}=0.250 \\
\mathrm{p}=0.091\end{array}$ & $\begin{array}{l}R=0.173 \\
p=0.245\end{array}$ & $\begin{array}{l}\mathrm{R}=0.130 \\
\mathrm{p}=0.383\end{array}$ & $\begin{array}{l}\mathrm{R}=0.235 \\
\mathrm{p}=0.111\end{array}$ & $\begin{array}{l}\mathrm{R}=0.212 \\
\mathrm{p}=0.153\end{array}$ & $\begin{array}{l}\mathrm{R}=0.146 \\
\mathrm{p}=0.328\end{array}$ & $\begin{array}{l}R=0.145 \\
p=0.330\end{array}$ & $\begin{array}{c}\mathrm{R}=-0.055 \\
\mathrm{p}=0.711\end{array}$ & $\begin{array}{l}\mathrm{R}=0.193 \\
\mathrm{p}=0.194\end{array}$ & $\begin{array}{l}\mathrm{R}=0.203 \\
\mathrm{p}=0.172\end{array}$ \\
\hline $\begin{array}{c}\text { CAT } \\
(29.90 \pm 17 \\
\text { 10l/mg protein })\end{array}$ & $\begin{array}{l}\mathrm{R}=0.263 \\
\mathrm{p}=0.074\end{array}$ & $\begin{array}{l}\mathrm{R}=0.337 \\
\mathrm{p}=0.020^{*}\end{array}$ & $\begin{array}{l}\mathrm{R}=0.203 \\
\mathrm{p}=0.171\end{array}$ & $\begin{array}{l}R=0.260 \\
p=0.078\end{array}$ & $\begin{array}{l}R=0.345 \\
p=0.018^{*}\end{array}$ & $\begin{array}{l}R=0.258 \\
p=0.080\end{array}$ & $\begin{array}{l}\mathrm{R}=0.221 \\
\mathrm{p}=0.136\end{array}$ & $\begin{array}{l}R=0.375 \\
p=0.009^{*}\end{array}$ & $\begin{array}{l}\mathrm{R}=0.281 \\
\mathrm{p}=0.055\end{array}$ & $\begin{array}{l}\mathrm{R}=0.326 \\
\mathrm{p}=0.025^{*}\end{array}$ \\
\hline $\begin{array}{c}\text { 8-OH-dG } \\
(421.86 \pm \\
59.67 \mathrm{Pg} / \mathrm{mL})\end{array}$ & $\begin{array}{l}\mathrm{R}=0.228 \\
\mathrm{p}=0.123\end{array}$ & $\begin{array}{l}\mathrm{R}=0.214 \\
\mathrm{p}=0.148\end{array}$ & $\begin{array}{l}\mathrm{R}=0.364 \\
\mathrm{p}=0.012^{*}\end{array}$ & $\begin{array}{l}\mathrm{R}=0.175 \\
\mathrm{p}=0.239\end{array}$ & $\begin{array}{l}\mathrm{R}=0.206 \\
\mathrm{p}=0.164\end{array}$ & $\begin{array}{l}\mathrm{R}=0.099 \\
\mathrm{p}=0.509\end{array}$ & $\begin{array}{l}\mathrm{R}=0.225 \\
\mathrm{p}=0.128\end{array}$ & $\begin{array}{l}\mathrm{R}=0.305 \\
\mathrm{p}=0.037^{*}\end{array}$ & $\begin{array}{l}\mathrm{R}=0.389 \\
\mathrm{p}=0.007^{*}\end{array}$ & $\begin{array}{l}R=0.280 \\
p=0.056\end{array}$ \\
\hline $\begin{array}{c}\text { GPx } \\
100.36 \pm 23.66 \\
\mathrm{nmol} / \mathrm{mg} \\
\text { protein) }\end{array}$ & $\begin{array}{c}\mathrm{R}=0.073 \\
0.626\end{array}$ & $\begin{array}{c}R=-0.081 \\
p=0.588\end{array}$ & $\begin{array}{l}\mathrm{R}=0.162 \\
\mathrm{p}=0.275\end{array}$ & $\begin{array}{l}R=0.058 \\
p=0.697\end{array}$ & $\begin{array}{l}R=0.048 \\
p=0.746\end{array}$ & $\begin{array}{c}\mathrm{R}=-0.111 \\
\mathrm{p}=0.457\end{array}$ & $\begin{array}{l}R=0.210 \\
p=0.157\end{array}$ & $\begin{array}{c}\mathrm{R}=0.038 \\
\mathrm{p}=0.8\end{array}$ & $\begin{array}{l}\mathrm{R}=0.203 \\
\mathrm{p}=0.170\end{array}$ & $\begin{array}{c}\mathrm{R}=0.085 \\
0.572\end{array}$ \\
\hline
\end{tabular}

Data represent mean \pm SD. ${ }^{*} \mathrm{p}<0.05$.

Table 3. The correlation between oxidative stress markers and neurocognitive impairment in radiology staff.

\begin{tabular}{|c|c|c|c|c|c|c|c|c|c|c|}
\hline & $\begin{array}{c}\text { PS (28.98 } \pm \\
3.49)\end{array}$ & $\begin{array}{c}\quad \text { IE (40.5 } \\
\quad \pm 6.28)\end{array}$ & $\begin{array}{l}\text { SA (36.43 } \\
\pm 7.05)\end{array}$ & $\begin{array}{c}\text { DA (16.83 } \\
\pm 2.24)\end{array}$ & $\begin{array}{l}\text { NVM (16.35 } \\
\quad \pm 2.82)\end{array}$ & $\begin{array}{c}\mathrm{PM}(28.63 \\
\pm 6.11)\end{array}$ & $\begin{array}{c}\text { VM } \\
(31 \pm 5.52)\end{array}$ & $\begin{array}{c}\mathrm{SF}(21.9 \pm \\
3.66)\end{array}$ & $\begin{array}{l} \pm \text { Sener (76.58 } \\
\quad \pm 11.63)\end{array}$ & $\begin{array}{cc}8 & \text { TOTAL } \\
(297.2 \pm 37.32)\end{array}$ \\
\hline $\begin{array}{c}\text { LPO } \\
(6.43 \pm 1.81 \\
\mu \mathrm{M})\end{array}$ & $\begin{array}{l}\mathrm{R}=0.031 \\
\mathrm{P}=0.847\end{array}$ & $\begin{aligned} \mathrm{R} & =-0.198 \\
\mathrm{p} & =0.220\end{aligned}$ & $\begin{array}{c}\mathrm{R}=-0.091 \\
\mathrm{p}=0.578\end{array}$ & $\begin{array}{c}\mathrm{R}=-0.144 \\
\mathrm{p}=0.376\end{array}$ & $\begin{array}{c}\mathrm{R}=-0.121 \\
\mathrm{p}=0.456\end{array}$ & $\begin{array}{c}\mathrm{R}=-0.014 \\
\mathrm{P}=0.933\end{array}$ & $\begin{array}{c}\mathrm{R}=-0.089 \\
\mathrm{p}=0.584\end{array}$ & $\begin{array}{l}\mathrm{R}=0.077 \\
\mathrm{p}=0.637\end{array}$ & $\begin{array}{c}R=-0.057 \\
p=0.728\end{array}$ & $\begin{array}{c}\mathrm{R}=-0.092 \\
\mathrm{p}=0.574\end{array}$ \\
\hline $\begin{array}{c}\text { MPO } \\
(7.94 \pm 9.36 \\
\mu \mathrm{M})\end{array}$ & $\begin{array}{l}\mathrm{R}=0.230 \\
\mathrm{p}=0.153\end{array}$ & $\begin{array}{l}\mathrm{R}=0.096 \\
\mathrm{p}=0.557\end{array}$ & $\begin{array}{l}\mathrm{R}=0.226 \\
\mathrm{p}=0.097\end{array}$ & $\begin{array}{l}\mathrm{R}=0.022 \\
\mathrm{p}=0.891\end{array}$ & $\begin{array}{l}\mathrm{R}=0.123 \\
\mathrm{p}=0.449\end{array}$ & $\begin{array}{l}\mathrm{R}=0.050 \\
\mathrm{p}=0.760\end{array}$ & $\begin{array}{l}\mathrm{R}=0.157 \\
\mathrm{p}=0.333\end{array}$ & $\begin{array}{l}\mathrm{R}=0.252 \\
\mathrm{p}=0.117\end{array}$ & $\begin{array}{l}R=0.108 \\
p=0.506\end{array}$ & $\begin{array}{l}\mathrm{R}=0.188 \\
\mathrm{p}=0.245\end{array}$ \\
\hline $\begin{array}{c}\text { SOD } \\
(0.65 \pm 0.29 \\
\mathrm{U} / \mathrm{mg} \\
\text { protein })\end{array}$ & $\begin{array}{l}\mathrm{R}=0.544 \\
\mathrm{P}=0.000^{*}\end{array}$ & $\begin{array}{l}\mathrm{R}=0.434 \\
\mathrm{p}=0.005^{*}\end{array}$ & $\begin{array}{l}\mathrm{R}=0.397 \\
\mathrm{p}=0.011^{*}\end{array}$ & $\begin{array}{l}\mathrm{R}=0.073 \\
\mathrm{p}=0.656\end{array}$ & $\begin{array}{l}\mathrm{R}=0.199 \\
\mathrm{p}=0.217\end{array}$ & $\begin{array}{l}\mathrm{R}=0.276 \\
\mathrm{p}=0.085\end{array}$ & $\begin{array}{l}\mathrm{R}=0.502 \\
\mathrm{p}=0.001^{*}\end{array}$ & $\begin{array}{l}\mathrm{R}=0.032 \\
\mathrm{p}=0.844\end{array}$ & $\begin{array}{l}\mathrm{R}=0.287 \\
\mathrm{p}=0.072\end{array}$ & $\begin{array}{l}\mathrm{R}=0.403 \\
\mathrm{p}=0.006^{*}\end{array}$ \\
\hline $\begin{array}{c}\text { CAT } \\
(29.90 \pm 17 \\
\mathrm{mol} / \mathrm{mg} \\
\text { protein })\end{array}$ & $\begin{array}{l}\mathrm{R}=0.269 \\
\mathrm{p}=0.093\end{array}$ & $\begin{array}{l}\mathrm{R}=0.114 \\
\mathrm{p}=0.484\end{array}$ & $\begin{array}{l}\mathrm{R}=0.101 \\
\mathrm{p}=0.536\end{array}$ & $\begin{array}{c}\mathrm{R}=-0.117 \\
\mathrm{p}=0.473\end{array}$ & $\begin{array}{c}\mathrm{R}=-0.209 \\
\mathrm{p}=0.196\end{array}$ & $\begin{array}{l}\mathrm{R}=0.157 \\
\mathrm{p}=0.333\end{array}$ & $\begin{array}{l}\mathrm{R}=0.016 \\
\mathrm{p}=0.924\end{array}$ & $\begin{array}{l}\mathrm{R}=0.139 \\
\mathrm{p}=0.393\end{array}$ & $\begin{array}{c}\mathrm{R}=-0.091 \\
\mathrm{p}=0.577\end{array}$ & $\begin{array}{l}\mathrm{R}=0.054 \\
\mathrm{p}=0.740\end{array}$ \\
\hline $\begin{array}{c}\text { 8-OH-dG } \\
(421.86 \pm \\
59.67 \mathrm{Pg} / \mathrm{mL})\end{array}$ & $\begin{array}{c}R=-0.031 \\
p=0.847\end{array}$ & $\begin{array}{l}\mathrm{R}=0.046 \\
\mathrm{p}=0.780\end{array}$ & $\begin{array}{c}\mathrm{R}=-0.043 \\
\mathrm{p}=0.793\end{array}$ & $\begin{array}{l}\mathrm{R}=0.062 \\
\mathrm{p}=0.706\end{array}$ & $\begin{array}{c}\mathrm{R}=-0.007 \\
\mathrm{p}=0.966\end{array}$ & $\begin{array}{c}\mathrm{R}=-0.042 \\
\mathrm{p}=0.795\end{array}$ & $\begin{array}{c}\mathrm{R}=-0.070 \\
\mathrm{p}=0.699\end{array}$ & $\begin{array}{l}\mathrm{R}=0.075 \\
\mathrm{p}=0.646\end{array}$ & $\begin{array}{l}\mathrm{R}=0.005 \\
\mathrm{p}=0.973\end{array}$ & $\begin{array}{c}\mathrm{R}=-0.008 \\
\mathrm{p}=0.959\end{array}$ \\
\hline $\begin{array}{c}\text { GPx } \\
(100.36 \pm \\
23.66 \\
\text { nmol/mg } \\
\text { protein })\end{array}$ & $\begin{aligned} R & =-0.61 \\
p & =0.70\end{aligned}$ & $\begin{array}{c}\mathrm{R}=0.000 \\
\mathrm{p}=1\end{array}$ & $\begin{array}{l}\mathrm{R}=0.028 \\
\mathrm{p}=0.863\end{array}$ & $\begin{array}{l}\mathrm{R}=0.131 \\
\mathrm{p}=0.421\end{array}$ & $\begin{array}{l}\mathrm{R}=0.103 \\
\mathrm{p}=0.526\end{array}$ & $\begin{array}{l}\mathrm{R}=0.238 \\
\mathrm{p}=0.139\end{array}$ & $\begin{aligned} \mathrm{R} & =0.140 \\
\mathrm{p} & =0.39\end{aligned}$ & $\begin{array}{l}\mathrm{R}=0.289 \\
\mathrm{p}=0.070\end{array}$ & $\begin{array}{l}R=0.136 \\
p=0.404\end{array}$ & $\begin{array}{l}\mathrm{R}=0.146 \\
\mathrm{p}=0.369\end{array}$ \\
\hline
\end{tabular}

Data represent mean \pm SD. ${ }^{*} \mathrm{p}<0.05$. 
ported a positive correlation between SOD on one side and PS $(R=0.544, p=0.000)$, IE $(R=0.434, p=0.005)$, SA $(\mathrm{R}=0.397, \mathrm{p}=0.011)$ and VM $(\mathrm{R}=0.502, \mathrm{p}=0.001)$ on the other side. There are not relationship between exposure history and psychological and cognitive state in workers (Table 4). However, the SOM, INT, DEP and PHOB were considerably different in male and female workers (see Table 5). At last, Table 6 has shown that the verbal memory (VM), nonverbal memory (NVM), prospective memory (PM) and total were significantly different in both male and female workers and no significant different in psychomotor speed (PS), selective attention (SA), divided attention (DA), spatial functioning (SF) and initiative/energy (IE).

\section{Discussion}

Findings of present study vividly showed a salient difference between male and female workers in symptoms of psychological disorders. Also there is positive correlationship between oxidative stress and cognitive neuropsychological disorders. These results are related to the conclusion of other researches done on the psychological disorders due to oxidative stress, for example, [6]-[19] [22] [28].

In other word, some studies have mentioned that exposure to radiation is as a reason of brain and cognitive disorders.

Animals and humans have a lot of problems because of exposure to radiation such as: neuronal inflammation, apoptosis, reduced oligodendrocytes and myelin sheath, damage to neurons [29] [30], down-regulation of neural pathways in mice [31], cognitive disorders caused by micro environmental factors including oxidative stress and inflammation [32], psychological stress [33] and emotional stress [34]. In some patients who cured with radio therapy, memory deficits [35], depression [36]. Social and psychological disorders [37] can be observed. This damage can be explained in different ways as follow.

Table 4. The relationship between exposure history and psychological and cognitive state in workers.

\begin{tabular}{|c|c|c|c|c|c|c|c|c|c|c|}
\hline & Somatization & $\begin{array}{l}\text { Obsessive- } \\
\text { Compulsive }\end{array}$ & $\begin{array}{c}\text { Interpersonal } \\
\text { Sensitivity }\end{array}$ & Depression & Anxiety & Hostility & $\begin{array}{l}\text { Phobic } \\
\text { Anxiety }\end{array}$ & $\begin{array}{l}\text { Paranoid } \\
\text { Ideation }\end{array}$ & Psychoticism & TOTAL \\
\hline $\begin{array}{l}\text { Work } \\
\text { History }\end{array}$ & & $\begin{array}{c}\mathrm{R}=-0.199 \\
\mathrm{p}=0.179\end{array}$ & $\begin{array}{c}\mathrm{R}=-0.004 \\
\mathrm{p}=0.980\end{array}$ & & & $\begin{array}{c}\mathrm{R}=-0.021 \\
\mathrm{p}=0.886\end{array}$ & & & $\begin{aligned} R & =-0.081 \\
p & =0.590\end{aligned}$ & \\
\hline & PS & IE & $\mathrm{S}$ & DA & NVM & PM & VM & SF & \multicolumn{2}{|c|}{ Total } \\
\hline $\begin{array}{l}\text { Work } \\
\text { History }\end{array}$ & $\begin{array}{c}R=-0.168 \\
p=0.3\end{array}$ & $\begin{array}{c}\mathrm{R}=-0.099 \\
\mathrm{p}=0.541\end{array}$ & $\begin{array}{c}\mathrm{R}=-0.116 \\
\mathrm{p}=0.476\end{array}$ & $\begin{array}{c}\mathrm{R}=-0.057 \\
\mathrm{p}=0.727\end{array}$ & $\begin{aligned} R & =-0.114 \\
p & =0.485\end{aligned}$ & $\begin{array}{c}\mathrm{R}=-0.117 \\
\mathrm{p}=0.470\end{array}$ & $\begin{aligned} R & =-0.305 \\
p & =0.056\end{aligned}$ & $\begin{array}{c}\mathrm{R}=-0.211 \\
\mathrm{p}=0.191\end{array}$ & \multicolumn{2}{|c|}{$\begin{array}{c}\mathrm{R}=-0.188 \\
\mathrm{p}=0.245\end{array}$} \\
\hline
\end{tabular}

Table 5. The status of psychological distress in male and female staff.

\begin{tabular}{ccccccccccc}
\hline Group Somatization & $\begin{array}{c}\text { Obsessive- Interpersonal } \\
\text { Compulsive }\end{array}$ & Sensitivity & Depression & Anxiety & Hostility & $\begin{array}{c}\text { Phobic } \\
\text { Anxiety }\end{array}$ & $\begin{array}{c}\text { Paranoid } \\
\text { Ideation }\end{array}$ & Psychoticism & Total \\
\hline Male & $0.59 \pm 0.64$ & $0.84 \pm 0.64$ & $0.53 \pm 3.77$ & $0.62 \pm 0.57$ & $0.53 \pm 0.58$ & $0.65 \pm 0.66$ & $0.43 \pm 0.46$ & $0.84 \pm 0.82$ & $0.41 \pm 0.51$ & $55.42 \pm 45.25$ \\
Female & $1 \pm 0.70$ & $1.1 \pm 0.73$ & $1.04 \pm 0.73$ & $1.03 \pm 0.75$ & $0.94 \pm 0.77$ & $0.69 \pm 0.57$ & $0.77 \pm 0.61$ & $1.24 \pm 0.70$ & $0.75 \pm 0.63$ & $88.04 \pm 51.52$ \\
p Value & $0.042^{*}$ & 0.220 & $0.013^{*}$ & $0.049^{*}$ & 0.052 & 0.835 & $0.044^{*}$ & 0.079 & 0.057 & $0.030^{*}$ \\
df & 1 & 1 & 1 & 1 & 1 & 1 & 1 & 1 & 1 & 1 \\
F & 4.36 & 1.54 & 6.73 & 4.1 & 4 & 0.044 & 4.3 & 3.24 & 3.81 & 5 \\
\hline
\end{tabular}

Data represent mean $\pm \mathrm{SD} .{ }^{*} \mathrm{p}<0.05$.

Table 6. The status of cognitive function in male and female staff.

\begin{tabular}{cccccccccc}
\hline Group & PS & SA & DA & VM & NVM & PM & SF & IE & Total \\
\hline Male & $29.94 \pm 3.98$ & $38.64 \pm 7.82$ & $16.76 \pm 2.54$ & $33.24 \pm 5.17$ & $17.76 \pm 2.54$ & $31.29 \pm 7.57$ & $22.24 \pm 4.94$ & $41.64 \pm 7.39310 .76 \pm 39.93$ \\
Female & $28.26 \pm 2.97$ & $34.78 \pm 6.09$ & $16.87 \pm 2.05$ & $29.35 \pm 5.29$ & $15.30 \pm 2.64$ & $26.65 \pm 3.86$ & $21.65 \pm 2.40$ & $39.65 \pm 5.33$ & $287.17 \pm 32.59$ \\
p Value & 0.134 & 0.087 & 0.886 & $0.026^{*}$ & $0.005^{*}$ & $0.015^{*}$ & 0.624 & 0.327 & $0.047^{*}$ \\
df & 1 & 1 & 1 & 1 & 1 & 1 & 1 & 1 & 1 \\
F & 2.34 & 3.09 & 0.021 & 5.39 & 9 & 6.43 & 0.244 & 0.986 & 4.23 \\
\hline
\end{tabular}

PS: psychomotor speed; SA: selective attention; DA: divided attention; VM: verbal memory; NVM: nonverbal memory; PM: prospective memory; SF: spatial functioning; I/E: initiative/energy. Data represent mean $\pm \mathrm{SD} .{ }^{*} \mathrm{p}<0.05$. 
Firstly, the limbic system that has duty for creating and understanding emotion since human consume more oxygen in this part of brain than others. As a result, high oxygen burning up raised the defenselessness to free radicals such as ROS. Secondly, free radicals will impact on neurotransmitters' in addition to damaging cell structures like mitochondria. Psychological activities can be controlled by most of neurotransmitters straightly. As an example, the chemical activity which produced from improved ROS can reduce serotonin and this may finish in depression. Thirdly the genetic constitution can cause to hurt. For example, if amygdala as a gene HTT- 5 that is responsible for controlling the activity of brain has been damaged, it will cause autism signs and social prevention. When symptoms of autism, severe anxiety and social phobia have been found, this presupposition is established.

It is worth mentioning that the subjects have chosen from hospital staff. Thus, the effects of other matters, including burn-out symptoms are not negligible. Job stress can create exhaustion, so it may make psychological symptoms worse. It can be imagined that the harm produced by oxidative stress accompany with job exhaustion cause mental disorders. Nutrition as one of environmental factors among hospital staff may be associated to oxidative stress and psychological factors. Furthermore, the influence of contextual factors in mental illnesses cannot be ignored.

The researchers noted that a holistic view should be taken into account in future research in order to study the relations of all the factors involved. The stability of these damages during time would be recommended. All in all, finding a moderating factor in the harsh effects of free radical son mental diseases may cause to realizing new interventions to improve patient care.

\section{Acknowledgements}

The researchers would be so thankful to all subjects and authorities of the Namazi hospital in Shiraz for their sincerely support. Authors thank assistance of Iran National Science Foundation (INSF) and support of Mohit Palayesh Arya Scientific Company.

\section{References}

[1] Malekirad, A.A., Ranjbar, A., Rahzani, K., Pilehvarian, A.A., Rezaie, A., Zamani, M.J., et al. (2005) Oxidative Stress in Radiology Staff. Environmental Toxicology and Pharmacology, 20, 215-218. http://dx.doi.org/10.1016/j.etap.2005.01.005

[2] Zeraatpishe, A., Oryan, S., Bagheri, M.H., Pilevarian, A.A., Malekirad, A.A., Baeeri, M., et al. (2011) Effects of Melissa officinalis L. on Oxidative Status and DNA Damage in Subjects Exposed to Long-Term Low-Dose Ionizing Radiation. Toxicology and Industrial Health, 27, 205-212. http://dx.doi.org/10.1177/0748233710383889

[3] Pal, R., Ahmed, T., Kumar, V., Suke, S.G., Ray, A. and Banerjee, B.D. (2009) Protective Effects of Different Antioxidants against Endosulfan-Induced Oxidative Stress and Immunotoxicity in Albino Rats. Indian Journal of Experimental Biology, 47, 723-729.

[4] Fani, A., Malekirad, A.A., Allahnazem, H., Rahzani, K., Ranjbar, A., Vosough-ghanbari, S., et al. (2008) On the Benefit of Cinnamomumzeylanicum for Radiology Unit Staff. Journal of Medical Sciences, 8, 384-389.

http://scialert.net/abstract/?doi=jms.2008.384.389

[5] Jokanović, M. and Kosanović, M. (2010) Neurotoxic Effects in Patients Poisoned with Organophosphorus Pesticides. Environmental Toxicology and Pharmacology, 29, 195-201. http://dx.doi.org/10.1016/j.etap.2010.01.006

[6] Tsaluchidu, S., Cocchi, M., Tonello, L. and Puri, B.K. (2008) Fatty Acids and Oxidative Stress in Psychiatric Disorders. BMC Psychiatry, 8, S5. http://dx.doi.org/10.1186/1471-244X-8-S1-S5

[7] Shao, L., Young, L.T. and Wang, J.F. (2005) Chronic Treatment with Mood Stabilizers Lithium and Valproate Prevents Excitotoxicity by Inhibiting Oxidative Stress in Rat Cerebral Cortical Cells. Biological Psychiatry, 58, 879-884. http://dx.doi.org/10.1016/j.biopsych.2005.04.052

[8] Bilici, M., Efe, H., Köroğlu, M.A., Uydu, H.A., Bekaroğlu, M. and Değer, O. (2001) Antioxidative Enzyme Activities and Lipid Peroxidation in Major Depression: Alterations by Antidepressant Treatments. Journal of Affective Disorders, 64, 43-51. http://dx.doi.org/10.1016/S0165-0327(00)00199-3

[9] Sarandol, A., Sarandol, E., Eker, S.S., Erdinc, S., Vatansever, E. and Kirli, S. (2007) Major Depressive Disorder Is Accompanied with Oxidative Stress: Short-Term Antidepressant Treatment Does Not Alter Oxidative-Antioxidative Systems. Human Psychopharmacology: Clinical and Experimental, 22, 67-73.

[10] Andreazza, A.C., Frey, B.N., Erdtmann, B., Salvador, M., Rombaldi, F., Santin, A., et al. (2007) DNA Damage in Bipolar Disorder. Psychiatry Research, 153, 27-32. http://dx.doi.org/10.1016/j.psychres.2006.03.025 
[11] Ersan, S., Bakir, S., Erdal Ersan, E. and Dogan, O. (2006) Examination of Free Radical Metabolism and Antioxidant Defence System Elements in Patients with Obsessive-Compulsive Disorder. Progress in Neuro-Psychopharmacology and Biological Psychiatry, 30, 1039-1042. http://dx.doi.org/10.1016/j.pnpbp.2006.03.034

[12] Chakraborty, S., Singh, O.P., Dasgupta, A., Mandal, N. and Nath Das, H. (2009) Correlation between Lipid Peroxidation-Induced TBARS Level and Disease Severity in Obsessive-Compulsive Disorder. Progress in Neuro-Psychopharmacology and Biological Psychiatry, 33, 363-366. http://dx.doi.org/10.1016/j.pnpbp.2009.01.001

[13] Ozdemir, E., Cetinkaya, S., Ersan, S., Kucukosman, S. and Ersan, E.E. (2009) Serum Selenium and Plasma Malon-Dialdehyde Levels and Antioxidant Enzyme Activities in Patients with Obsessive-Compulsive Disorder. Progress in NeuroPsychopharmacology and Biological Psychiatry, 33, 62-65. http://dx.doi.org/10.1016/j.pnpbp.2008.10.004

[14] Ratnakar, S., Banupriya, C., Doureradjou, P., Vivekanandam, S., Srivastava, M.K. and Koner, B.C. (2008) Evaluation of Anxiety, Depression and Urinary Protein Excretion among the Family Caregivers of Advanced Cancer Patients. Biological Psychology, 79, 234-238. http://dx.doi.org/10.1016/j.biopsycho.2008.06.001

[15] Forlenza, M.J. and Miller, G.E. (2006) Increased Serum Levels of 8-Hydroxy-2-Deoxyguanosine in Clinical Depression. Psychosomatic Medicine, 68, 1-7. http://dx.doi.org/10.1097/01.psy.0000195780.37277.2a

[16] Söğüt, S., Zoroğlu, S.S., Ozyurt, H., Yilmaz, H.R., Ozuğurlu, F., Sivasli, E., Yetkin, Ö., Yanık, M., Tutkun, H., Savaş, H.A., Tarakçığlu, M. and Akyol, Ö. (2003) Changes in Nitric Oxide Levels and Antioxidant Activities May Have a Role in the Pathophysiological Mechanisms Involved in Autism. Clinica Chimica Acta, 331, 111-117. http://dx.doi.org/10.1016/S0009-8981(03)00119-0

[17] Chauhan, A., Chauhan, V., Brown, W.T. and Cohen, I. (2004) Oxidative Stress in Autism: Increased Lipid Peroxidation and Reduced Serum Levels of Ceruloplasmin and Transferring-The Antioxidant Proteins. Life Sciences, 75, 2539-2549. http://dx.doi.org/10.1016/j.lfs.2004.04.038

[18] Markesbery, W.R. and Lovell, M.A. (2007) Damage to Lipids, Proteins, DNA, and RNA in Mild Cognitive Impairment. JAMA Neurology, 64, 954-956. http://dx.doi.org/10.1001/archneur.64.7.954

[19] Gil, P., Fariñas, F., Casado, A. and López-Fernández, E. (2002) Malondialdehyde: A Possible Marker of Ageing. Gerontology, 48, 209-214. http://dx.doi.org/10.1159/000058352

[20] Derogatis, L.R., Lipman, R.S. and Cavi, L. (1973) SCL-90: An Outpatient Psychiatric Rating Scale-Preliminary Report. Psychopharmacology Bulletin, 9, 13-28.

[21] Bayrami, M., Hashemi, T., Malekirad, A.A., Ashayeri, H., Faraji, F. and Abdollahi, M. (2012) Electroencephalogram, Cognitive State, Psychological Disorders, Clinical Symptom, and Oxidative Stress in Horticulture Farmers Exposed to Organophosphate Pesticides. Toxicology and Industrial Health, 28, 90-96. http://dx.doi.org/10.1177/0748233711407243

[22] Malekirad, A.A., Mirabdollahi, M., Pilevarian, A.A., Nassajpour, A.R. and Abdollahi, M. (2013) Neurocognitive Impairment, Clinical and Biochemical Toxicity Biomarkers in Iron-Steel Workers. Toxicology and Industrial Health.

[23] Zimmermann, P., Messner, C., Poser, U. and Sedelmeier, P. (1991) Ein Fragebogen erlebter Defizite der Aufmerksamkeit (FEDA) [A Questionnaire Measuring Self-Experienced Deficits of Attention]. PsychologischesInstitut der Universität Freiburg, Freiburg.

[24] Moritz, S., Ferahli, S. and Naber, D. (2004) Memory and Attention Performance in Psychiatric Patients Lack of Correspondence between Clinician-Rated and Patient-Rated Functioning with Neuropsychological Test Results. Journal of the International Neuropsychological Society, 10, 623-633. http://dx.doi.org/10.1017/S1355617704104153

[25] Esterabeur, H. and Cheeseman, K. (1990) Determination of Aldehyids Lipid Peroxidation Products: Malondealdhyde and 4-Hydroxylnonenal. Methods in Enzymology, 186, 407-421. http://dx.doi.org/10.1016/0076-6879(90)86134-H

[26] Benzi, I.F. and Strain, S. (1999) Ferric Reducing Antioxidant Assay. Methods in Enzymology, 292, 15-27.

[27] Hu, M.L. and Dillard, C.J. (1994) Plasma SH and GSH Measurement. Methods in Enzymology, 233, 385-387.

[28] Ozcan, M.E., Gulec, M., Ozerol, E., Polat, R. and Akyol, O. (2004) Antioxidant Enzyme Activities and Oxidative Stress in Affective Disorders. International Clinical Psychopharmacology, 19, 89-95. http://dx.doi.org/10.1097/00004850-200403000-00006

[29] Marazziti, D., Baroni, S., Catena-Dell’Osso, M., Schiavi, E., Ceresoli, D., Conversano, C., Dell’Osso, L. and Picano, E. (2012) Cognitive, Psychological and Psychiatric Effects of Ionizing Radiation Exposure. Current Medicinal Chemistry, 19, 1864-1869. http://dx.doi.org/10.2174/092986712800099776

[30] Panagiotakos, G., Alshamy, G., Chan, B., Abrams, R., Greenberg, E., Saxena, A., Bradbury, M., Edgar, M., Gutin, P. and Tabar, V. (2007) Long-Term Impact of Radiation on the Stem Cell and Oligodendrocyte Precursors in the Brain. PLOS ONE, 2, Article ID: e588. http://dx.doi.org/10.1371/journal.pone.0000588

[31] Lowe, X.R., Bhattacharya, S., Marchetti, F. and Wyrobek, A.J. (2009) Early Brain Response to Low-Dose Radiation Exposure Involves Molecular Networks and Pathways Associated with Cognitive Functions, Advanced Aging and Alzheimer's Disease. Radiation Research, 171, 53-65. http://dx.doi.org/10.1667/RR1389.1 
[32] Fike, J.R. (2011) Physiopathology of Radiation-Induced Neurotoxicity. Revue Neurologique (Paris), 167, 746-750. http://dx.doi.org/10.1016/j.neurol.2011.07.005

[33] Cwikel, J.G., Gidron, Y. and Quastel, M. (2010) Low-Dose Environmental Radiation, DNA Damage, and Cancer: The Possible Contribution of Psychological Factors. Psychology, Health \& Medicine, 15, 1-16. http://dx.doi.org/10.1080/13548500903431493

[34] Moroz, B.B., Deshevoílu, B., Voronina, T.A., Lyrshchikova, A.V. and Lebedev, V.G. (2007) Effect of Mexidol on Hemopoietic System in Conditions of an Emotional Stress after Exposure to Ionizing Radiation. Radiatsionnaia Biologiia, Radioecologiia, 47, 163-170.

[35] Armstrong, C.L., Corn, B.W., Ruffer, J.E., Pruitt, A.A., Mollman, J.E. and Phillips, P.C. (2000) Radiotherapeutic Effects on Brain Function: Double Dissociation of Memory Systems. Neuropsychiatry, Neuropsychology, and Behavioral Neurology, 13, 101-111.

[36] Chen, S.C., Lai, Y.H., Liao, C.T., Lin, C.C. and Chang, J.T. (2010) Changes of Symptoms and Depression in Oral Cavity Cancer Patients Receiving Radiation Therapy. Oral Oncology, 46, 509-513. http://dx.doi.org/10.1016/j.oraloncology.2010.02.024

[37] Yen, P.N., Yang, C.C., Chang, P.W., Hwang, J.S., Lee, H.C., Kuo, K.L. and Lin, I.F. (2011) Perception of Quality of Life of a Cohort Population Years after Relocation from Previous Low-Dose Radiation Exposure in Co-60 Contaminated Buildings in Taiwan. International Journal of Radiation Biology, 87, 453-460. http://dx.doi.org/10.3109/09553002.2011.544372 


\section{Abbreviation}

Reactive Oxygen Species (ROS)

Nitrogen Reactive Species (RNS)

Bipolar Disorder (BD)

Social Phobia (SP)

Obsessive-Compulsive Disorder (OCD)

Tris Base, Tetraethoxy Propane (MDA)

2-Thiobarbituric Acid (TBA)

Trichloroacetic Acid (TCA)

Dithiobis-2-Nitrobenzoic Acid (DTNB)

2,4,6-Tripyridyl-Striazine (TPTZ)

Somatization (SOM)

Obsessive-Compulsive (OBS)

Hostility (HOS)

Interpersonal Sensitivity (INT)

Depression (DEP)

Psychoticism (PSY)

Anxiety (ANX)

Phobic Anxiety (PHOB)

Paranoid Ideation (PAR)

Global Severity Index (GSI)

Positive Symptom Distress Index (PSDI)

Positive Symptom Total (PST)

Glutathione Peroxidase (GPX)

Superoxide Dismutase (SOD)

8-Hydroxy-2-Deoxy Guano Sine (8-OH-dG)

Catalase (CAT)

Meyleoperoxidase (MPO)

Lipid Peroxidation (LPO)

Total Antioxidant Capacity (TAC)

Total Thiol Molecules (TTM)

Verbal Memory (VM)

Nonverbal Memory (NVM)

Prospective Memory (PM)

Psychomotor Speed (PS)

Selective Attention (SA)

Divided Attention (DA)

Spatial Functioning (SF)

Initiative/Energy (IE) 
Scientific Research Publishing (SCIRP) is one of the largest Open Access journal publishers. It is currently publishing more than 200 open access, online, peer-reviewed journals covering a wide range of academic disciplines. SCIRP serves the worldwide academic communities and contributes to the progress and application of science with its publication.

Other selected journals from SCIRP are listed as below. Submit your manuscript to us via either submit@scirp.org or Online Submission Portal.
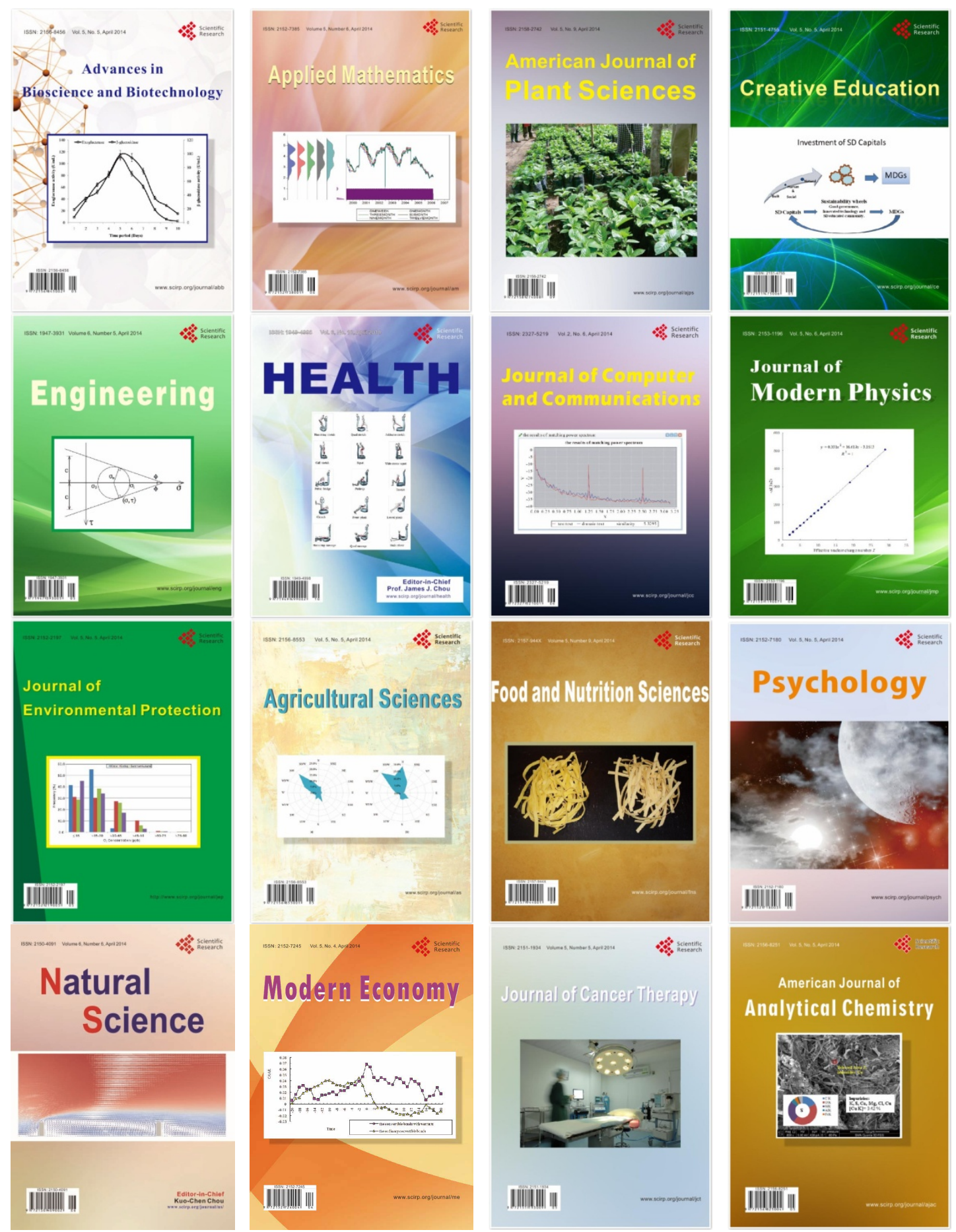